\title{
Investigating the Potential Effect of Consanguinity on Type 2 Diabetes Susceptibility in a Saudi Population
}

\author{
Ibrahim M. Gosadi ${ }^{a}$ Elizabeth C. Goyder ${ }^{b}$ Marion D. Teare ${ }^{b}$

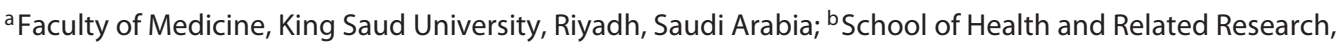 \\ Sheffield University, Sheffield, UK
}

\section{Key Words}

Type 2 diabetes · Inbreeding · Consanguinity · Genetics .

Saudi population

\begin{abstract}
Objective: To examine mechanisms by which consanguinity might increase the risk of type 2 diabetes (T2D) in a Saudi population. Methodology: 362 adult male participants were recruited, 179 were T2D patients and 183 healthy siblings. T2D severity was assessed in patients by recording age at diagnosis. In healthy subjects, diabetes risk was studied by measuring the body mass index, fasting blood glucose (FBG) level, and waist circumference. Extended pedigrees were constructed to calculate inbreeding coefficients. To account for tribal relatedness degrees reported in the constructed pedigrees, assumed inbreeding coefficients for tribal relatedness were added to the calculated inbreeding coefficients. A total of 23 SNPs associated with a higher risk of T2D were genotyped. Results: A significant inverse association was detected between inbreeding coefficients and age at diagnosis (Spearman's coefficient: $-0.186, p=0.013$ ). In 42 families, we were able to recruit 2 healthy siblings. Pearson's correlation coefficient of FBG between siblings was $0.317(p=0.04)$. The correlation between the siblings' FBG increased with an increasing degree of consanguinity. The effect of consanguin-
\end{abstract}

ity on the FBG level was further assessed by regression line analysis and by controlling for differences in age, caloric intake, and level of physical activity ( $\beta$ : $-0.118, p=0.024)$. No significant association between the number of T2D risk alleles and the traits was found. Conclusion: Our findings suggest that consanguinity might increase the risk of T2D by an earlier onset of the disease and by strengthening possible genetic effects on FBG.

(c) 2014 S. Karger AG, Basel

\section{Introduction}

Type 2 diabetes (T2D) is a public health concern in Saudi Arabia. According to the International Diabetes Federation, Saudi Arabia is among the top 10 countries for the prevalence of T2D across the globe [1]. Several epidemiological studies have been conducted in Saudi Arabia to estimate the prevalence of diabetes [2-8]. A review of these epidemiological studies found an increase in the prevalence of diabetes from $2.5 \%$ in 1982 to $23.7 \%$ in 2004 [9]. A more recent study reported that the prevalence of diabetes in Saudi Arabia was 34.1\% in males and $27.6 \%$ in females [8]. This profound increase in cases with diabetes is likely to be related to several environmental and genetic factors. These factors appear to make the Sau- 
di population more susceptible to the disease than other populations.

Several genetic variants have been suggested to increase the risk of T2D with different levels of effect, though most of the detected variants are presumed to act additively [10]. However, several epidemiological investigations were able to find an association between consanguinity and the risk of developing T2D [11-13]. A study conducted in Qatar reported higher odds of developing T2D among consanguineous marriages of first-degree cousins $(\mathrm{OR}=1.59,95 \% \mathrm{CI}=1.11-2.29)$. Furthermore, the odds of developing T2D among consanguineous marriages of second-degree cousins was smaller but statistically non-significant compared to marriages between first-degree cousins $(\mathrm{OR}=1.26,95 \% \mathrm{CI}=0.78-2.02)$ [11].

Another study conducted in Saudi Arabia revealed that a positive family history of T2D is more likely to be reported in families with consanguinity than in families without consanguinity $(\mathrm{OR}=13.4,95 \% \mathrm{CI}=8.64-23.98)$ [12]. However, this study only reported the odds of having a family history of T2D and did not actually provide which effect a history of consanguinity has on the T2D disease status. Finding an association between consanguinity and a higher risk of developing T2D might suggest possible recessive effects which increase the risk of developing T2D.

The power to detect recessive effects should be higher in the Saudi population due to the high level of consanguineous marriages. Saudi Arabia has a high prevalence of consanguinity compared to Western societies. Several investigations revealed that the percentage of consanguineous marriages ranges between $54 \%$ and $57 \%$, and most of these marriages are between first-degree cousins [14-16]. These studies covered various regions in Saudi Arabia and their sample sizes varied between 3,212 and 11,554 families.

The prevalence of consanguinity depends on several traditional and social factors. Families in Saudi Arabia are adherent to cultural behaviour, and marriage between first-degree cousins is almost the norm. It has been argued that marriage between relatives is more likely to be easily mediated, as mating individuals are brought up together in the same environment. Furthermore, such arrangements may ease spousal adjustment after marriage and enhance the stability of marriages. However, other economic reasons have been suggested, since marriage between relatives is a method of keeping financial assets and properties within the same family or tribe [14].

Higher consanguinity levels increase the overall homozygosity in a population. Increased homozygosity in- creases the power to detect recessive effects whether on monogenic illnesses or even more complex diseases such as T2D. Based on the available evidence, a synergism between T2D and inbreeding can be suspected. Recognizing how inbreeding increases the risk of T2D is an important step in strengthening the evidence. However, there is no evidence uncovering how inbreeding might lead to an increased risk of T2D.

Due to the multigenic nature of T2D, inheriting more risk alleles has been found to increase the risk [17]. At each locus, one might inherit a single or two copies of a risk allele. Additive models predict that those who inherit two copies of risk alleles at each locus will be at higher risk of developing T2D than those who inherit a single risk allele at each locus. Since inbreeding increases the chance of inheriting identical alleles at loci, it can also increase the risk of inheriting two identical copies of particular risk alleles at each locus and thus increase the risk of T2D. Additionally, higher consanguinity levels might enable to detect the possible recessive effect of T2D risk alleles.

The aim of this study was to examine the possible mechanisms by which inbreeding might increase the risk of T2D in a Saudi population. Since we have sampled affected subjects, we have used age at diagnosis as a marker of T2D severity. The study hypothesized a possible cumulative recessive effect on age at diagnosis of T2D. Additionally, an increased body mass index (BMI), elevated blood glucose levels, and central obesity are known to be risk factors for T2D. Part of the variation of these continuous traits can be attributed to population genetic variation at loci which influence the expression of these phenotypes. We can hypothesize that inbreeding can help in aggregating risk alleles inducing possible cumulative recessive effects, leading to the elevation of these risk factors, and subsequently the increase of T2D risk. In addition to measuring genetic variation between participants, environmental variation was measured using food frequency types and physical activity questionnaires to aid in better understanding the effect of variation in genotypes and environment on variation of traits related to T2D.

\section{Methodology}

\section{Study Participants}

The recruitment of subjects took place in the Jazan region, Saudi Arabia. T2D patients were identified and invited to participate through targeted primary health care centres (PHCs) with specialized diabetes clinics. The recruitment of healthy re- 
lated control participants was conducted during home visits. Data collection was initiated in July 2012 and completed in January 2013. Ethical approvals were obtained from the Directory of Health in Jazan region, Saudi Arabia, and the School of Health and Related Research, University of Sheffield, Sheffield, UK.

To be eligible for the study, the patients had to have available healthy siblings for recruitment. Patients with no available healthy siblings were recruited only if they had multiple siblings affected with T2D. The last criterion was established to avoid selection bias, since several families were found to have many affected siblings with T2D and no healthy siblings. The identification and approaching of cases was performed by PHC staff, and the recruitment was performed by one of the investigators (I.M.G.). All subjects were older than 18 years when recruited.

362 adult male participants were recruited, 179 of these were T2D patients and 183 were healthy participants. Confirmation of the disease status was based on the diagnosis made by medical practitioners. Patients with type 1 diabetes were excluded. All recruited healthy members were full siblings of the recruited patients and self-reportedly not diagnosed with T2D at the time of recruitment. During home visits, the patients were asked to provide a full family history so that consanguinity could be assessed and to complete dietary and physical activity questionnaires. The healthy siblings had their BMI, fasting blood glucose (FBG) level, and waist circumference measured in addition to completing the questionnaires.

\section{Data Collection}

Data collection was performed by a single investigator, and the same data collection tools were utilized for all participants. All data were collected during interviews. Extended pedigrees were constructed during interviews to aid in the calculation of inbreeding coefficients. All patients were asked about their age at diagnosis with T2D. The body weight of healthy participants was measured using the Beurer PS05 scale while the participants were wearing light clothing. Height was measured using the Seca 213 portable stadiometer. Waist circumference was measured using a measuring tape according to the regulations of the Joint British Societies' guidelines [18]. The measurements of blood glucose were made using the Optium Xceed glucometer. Before performing a measurement of blood glucose, we ensured that the participants had been fasting for the preceding $8 \mathrm{~h}$.

We used buccal swabs (SK-1S DNA Swab Pack-Isohelix) to collect DNA samples. Preservative capsules (SGC Dri-Capsules-Isohelix) were used to store the buccal swab samples. This method allowed storing the samples at normal room temperature. The process of DNA extraction and genotyping was performed by K-biosciences, Hoddesdon, UK, using the KASP (competitive allelespecific PCR) genotyping method. Genotyping success varied between 91 and $95 \%$ per SNP. To avoid measurement bias due to variations in the number of successfully genotyped SNPs, we eliminated participants with 3 or more SNPs that failed genotyping.

To account for environmental variation between participants, interviews were performed with all participants that included questionnaires on frequency of different food types and physical activity. These were a modified version of the questionnaires provided by the European Prospective Investigation into Cancer and Nutrition (EPIC) study. Modifications were performed to allow using the questionnaires on Saudi subjects.

Potential Effect of Consanguinity on T2D

Susceptibility in a Saudi Population

\section{Calculation of Inbreeding Coefficients}

The calculations of inbreeding coefficients were based on drawn pedigrees. An inbreeding coefficient measures the probability of having two alleles that are identical by descent from a single common ancestor. One of the methods of calculating inbreeding coefficients is called the path method [19]. This method relies on the number of parents traced from an individual to a shared common ancestor. Using the path method, inbreeding coefficients can be calculated by using the following formula:

$$
F x=\sum_{i=1}^{j}\left[\left(\frac{1}{2}\right)^{n_{i}}\left(1+F A_{i}\right)\right] .
$$

where $F x$ is the inbreeding coefficient of the individual, who has $j$ common ancestors; $n_{i}$ is the number of pedigree members connecting the individual and the $i$-th common ancestor, and $F A_{i}$ is the inbreeding coefficient of the $i$-th common ancestor.

Several levels of consanguinity were observed during data collection. As expected, we detected marriages of unrelated couples, first cousins, second cousins, third cousins, and marriages between individuals and their parental cousins. However, we also observed two other forms of relatedness that should be considered during the calculation of inbreeding coefficients: marriages between individuals who are from the same tribe or subtribe.

Tribe and subtribe are very important cultural concepts in Saudi Arabia. Tribal relatedness is a valued concept in Saudi Arabia and in many instances it leads to differentiations between Saudi people. These differentiations could be based on geographic, economic, political, and social factors. Due to these factors, tribes tend to be distinguished from each other and, eventually, individuals tend to get married to people from the same tribe.

Due to religious and cultural beliefs, Saudi families tend to have larger kinships compared to other societies. After several generations, a small tribe starts to grow and, due to the differentiation factors, subtribes begin to develop, leading to further differentiations of members of the same tribe. On average, a subtribe is composed of hundreds of individuals, and several subtribes make a bigger tribe composed of thousands of individuals.

Since Saudi Arabia has witnessed a high level of consanguinity, multiple lines of descent are expected to occur. Most of the interviewed individuals were able to report a shared parental common ancestor if their parents were first, second, or third cousins. However, we believe that the real inbreeding coefficients are very likely to be augmented by multiple lines of descent induced by common ancestors from shared tribes or subtribes.

Most of the studies which utilized pedigrees to calculate inbreeding coefficients reported parental relatedness as a categorical variable. In most of these situations, the parents were reported to be first or second cousins or unrelated. However, we believe that utilizing this approach in a Saudi population is likely to neglect the long-standing effect of tribal relatedness. In several situations, parents would be described as unrelated where, in fact, they could be related through several lines of descent through shared subtribes or tribes. The concept of incorporating distant tribal relatedness when calculating inbreeding coefficients is new, and, according to our knowledge, no other studies have attempted to do this.

To account for the possible effect of tribal relatedness on the calculated inbreeding coefficients, we used shared tribe or subtribe as two new concepts when drawing pedigrees. This degree of relatedness was described when the participant interviewed did not ex- 


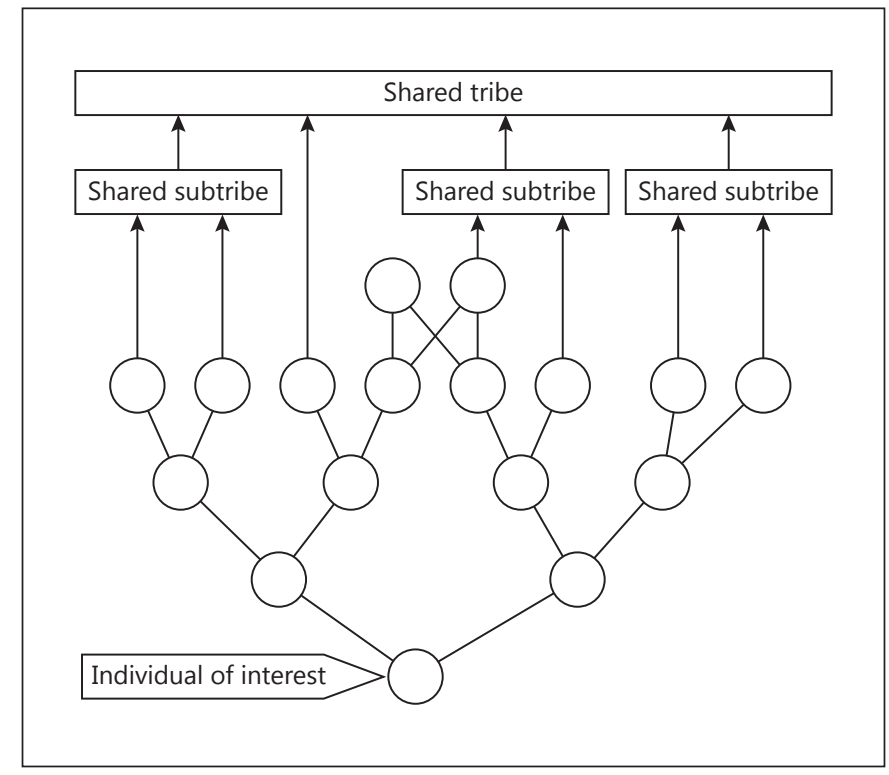

Fig. 1. Pedigree drawn to illustrate different degrees of relatedness in marriages.

actly know who the distant common ancestors were. Additionally, many subjects were able to report a shared common ancestor when their parents were first, second, or third cousins, and they were also asked whether their distant grandfathers were related through shared tribes or subtribes. Therefore, reported withintribe or -subtribe marriages were included in all pedigrees drawn.

As subjects reported that their parents belonged to the same tribe or subtribe, this obviously implied that there is a common ancestor but the exact relation was not known. Figure 1 is an example of a tribe structure observed during data collection. The level of consanguinity that occurred between parents and grandparents of subjects of interest can easily be observed, and how this might have affected the overall calculated inbreeding coefficient.

Since there are no formal genealogical records in Saudi Arabia, we had to rely on the pedigrees constructed during the interviews to calculate inbreeding coefficients. In all constructed pedigrees, the most distant reported common ancestor was when the parents of the interviewed participants were third cousins. Additionally, all interviewed participants were able to report the degree of tribal relatedness. However, without genomic measurements, it is impossible to estimate inbreeding coefficients for the mating of individuals from the same subtribe or the same tribe.

Since the most distant reported common ancestor was of parents who were third cousins, we could assume that parents who shared a subtribe or a tribe would be more distantly related than third cousins. Therefore, it seemed reasonable to assume that a mating between two members of a subtribe is equivalent to that between fifth-degree cousins, and that a mating between two members of a tribe is equivalent to that between seventh-degree cousins. Although these assumptions are arbitrary to some extent, they make a minimal contribution to the inbreeding coefficient, while taking all possible multiple lines of shared distant ancestors into consideration.
Asking the interviewees about the degree of parental relatedness went through several steps. In situations where the parents of the interviewed participants were cousins, further questions were asked to determine who the common ancestor was. After detecting the common ancestor, further information was collected about the degree of tribal relatedness of other grandparents, to consider other more distant lines of descent. If the participants were only able to reveal that their parents were distantly related, the investigator would ask the participants whether their grandparents were related through shared subtribes or tribes. Different degrees of tribal relatedness between grandparents were considered in each pedigree drawn, as shown in figure 1. Within the same pedigree, greatgrandparents could be unrelated, share a tribe, or a subtribe. These variations in the degree of relatedness of more distant grandparents are likely to increase the overall variation of calculated inbreeding coefficients.

In situations where a common ancestor was reported during pedigree construction, the estimated inbreeding coefficients were calculated by adding the values obtained from the tribal relatedness information to the inbreeding coefficient based on the known shared common ancestor. The pedigree illustrated in figure $1 \mathrm{ex}-$ plains how inbreeding coefficients are calculated when utilising the new concept introduced here. The parents of the individual of interest share a known common ancestor and are second cousins. In addition, there is a complicated network of relationships where several great-grandparents belong to the same subtribe or tribe.

The inbreeding coefficient of the individual of interest in figure 1 was calculated through several steps. Firstly, 15 pathways are related to the shared tribe, where each pathway contributes $0.001 \%$ to the overall inbreeding coefficient. Secondly, there is one pathway related to the shared subtribe which contributes $0.02 \%$ to the overall inbreeding coefficient. Thirdly, two pathways are related to a degree of second cousins which contribute $1.56 \%$.

The final estimation of the inbreeding coefficient is the result of combining the contributions made by all of the different pathways. The inbreeding coefficient when parents are second cousins is $1.56 \%$, but once all of the other distant pathways depicted in figure 1 are considered, the coefficient increases to $1.59 \%$. This is an estimation, but it takes into consideration other pathways that are difficult to ascertain precisely, such as a common ancestor in a subtribe or a tribe. Moreover, this estimation is entirely based on the classic path method with the only modification being a simple addition of the assumed values of tribal relatedness information.

\section{Selecting T2D SNP Data}

During the time of conducting this study, to our knowledge, there were no other studies which had performed a genetic association or replication studies in Saudi Arabia concerning T2D yet. Therefore, we could not know whether the T2D risk alleles which had been detected in European and Asian populations would be replicable in a Saudi population. Although about 60 loci have been found to be associated with T2D [10], this study was only able to genotype 23 SNPs due to a limited budget.

A review of the literature was performed to select those SNPs incurring a higher risk of T2D. SNP selection was based on several criteria. Firstly, selected SNPs had to be replicated in more than one population. Secondly, selected SNPs had to have been shown to affect more than one continuous trait related to T2D. Thirdly, selected SNPs had to be associated with a reduction of birth weight, since a reduced birth weight has been reported as a 
Table 1. List of genotyped SNPs and reason(s) for selecting each SNP

\begin{tabular}{|c|c|c|c|c|c|c|}
\hline No. & Marker & Locus & Chr & Allele $^{a}$ & Reason(s) for selection & Reference \\
\hline 1 & rs7903146 & TCF7L2 & 10 & $\mathrm{~T} / \mathrm{C}$ & $\begin{array}{l}\text { Most replicated among ethnicities } \\
\text { Affects FBG, insulin, and HbA1(c) levels }\end{array}$ & {$[21-23]$} \\
\hline 2 & rs13266634 & SLC30A8 & 8 & $\mathrm{C} / \mathrm{T}$ & $\begin{array}{l}\text { Replicated in European and Asian populations } \\
\text { Affects FBG, insulin, and HbA1(c) levels }\end{array}$ & {$[22,24-27]$} \\
\hline 3 & rs1111875 & HHEX & 10 & $\mathrm{C} / \mathrm{T}$ & $\begin{array}{l}\text { Replicated in European and Asian populations } \\
\text { Associated with a reduction in birth weight }\end{array}$ & {$[24,28-30]$} \\
\hline 4 & rs10811661 & CDKN2B & 9 & $\mathrm{~T} / \mathrm{C}$ & Replicated in European and Asian populations & {$[24,25,28,29,31]$} \\
\hline 5 & rs7754840 & CDKAL1 & 6 & $\mathrm{C} / \mathrm{G}$ & $\begin{array}{l}\text { Replicated in European, Asian, and Arab Lebanese } \\
\text { populations }\end{array}$ & {$[24,25,28,29,32]$} \\
\hline 6 & rs7756992 & CDKAL1 & 6 & $\mathrm{G} / \mathrm{C}$ & $\begin{array}{l}\text { Replicated in European, Asian and Arab Lebanese } \\
\text { populations } \\
\text { Associated with a reduction in birth weight }\end{array}$ & {$[26,29,30,32,33]$} \\
\hline 7 & rs10946398 & CDKAL1 & 6 & $\mathrm{~A} / \mathrm{C}$ & Replicated in European and Asian populations & {$[29,31]$} \\
\hline 8 & rs2237895 & KCNQ1 & 11 & $\mathrm{C} / \mathrm{T}$ & Replicated in European and Asian populations & {$[34,35]$} \\
\hline 9 & rs231362 & KCNQ1 & 11 & G/A & Replicated in European and Asian populations & {$[36,37]$} \\
\hline 10 & rs2237897 & KCNQ1 & 11 & $\mathrm{C} / \mathrm{T}$ & Replicated in European and Asian populations & [35] \\
\hline 11 & rs560887 & G6PC2 & 2 & $\mathrm{C} / \mathrm{T}$ & Affects FBG, insulin, and HbA1(c) levels & {$[17,22]$} \\
\hline 12 & rs10830963 & MTNR1B & 11 & $\mathrm{G} / \mathrm{C}$ & Affects FBG, insulin, and HbA1(c) levels & {$[17,22,38]$} \\
\hline 13 & rs1387153 & MTNR1B & 11 & $\mathrm{~T} / \mathrm{C}$ & Affects FBG and HbA1(c) levels & {$[38,39]$} \\
\hline$\overline{14}$ & rs4607517 & GCK & 7 & $\mathrm{~A} / \mathrm{G}$ & $\begin{array}{l}\text { Affects FBG, insulin, and HbA1(c) levels } \\
\text { Shared with new natal diabetes and MODY }\end{array}$ & {$[22,40]$} \\
\hline 15 & rs1799884 & GCK & 7 & $\mathrm{~T} / \mathrm{C}$ & Affects FBG and HbA1(c) levels & {$[38,39]$} \\
\hline 16 & rs780094 & GCKR & 2 & $\mathrm{C} / \mathrm{T}$ & Affects FBG and insulin levels & {$[22]$} \\
\hline 17 & rs11708067 & ADCY5 & 3 & $\mathrm{~A} / \mathrm{G}$ & $\begin{array}{l}\text { Affects FBG and insulin levels } \\
\text { Associated with a reduction in birth weight }\end{array}$ & {$[22,30]$} \\
\hline 18 & rs2191349 & $\begin{array}{l}\text { DGKB- } \\
\text { TMEM195 }\end{array}$ & 7 & $\mathrm{~T} / \mathrm{G}$ & Affects FBG and insulin levels & {$[22]$} \\
\hline 19 & rs174550 & FADS1 & 11 & $\mathrm{~T} / \mathrm{C}$ & Affects FBG and insulin levels & {$[22]$} \\
\hline 20 & rs7034200 & GLIS3 & 9 & $\mathrm{~A} / \mathrm{C}$ & Affects FBG and insulin levels & {$[22]$} \\
\hline 21 & rs5219 & KCNJ11 & 11 & $\mathrm{~T} / \mathrm{C}$ & $\begin{array}{l}\text { Shared with neonatal diabetes } \\
\text { Associated with a reduction in birth weight }\end{array}$ & {$[41,42]$} \\
\hline 22 & rs7957197 & HNF1a & 12 & $\mathrm{~T} / \mathrm{A}$ & Shared with MODY 3 & {$[36,43]$} \\
\hline$\overline{23}$ & Rs757210 & HNF1B & 17 & $\mathrm{~A} / \mathrm{G}$ & Shared with MODY 5 & {$[36,44]$} \\
\hline
\end{tabular}

Chr $=$ Chromosome. ${ }^{\text {a }}$ Alles are given as having effect/other.

risk factor for T2D [20]. Finally, SNPs had to have been found to be shared with other forms of monogenic diabetes to be genotyped. A list of genotyped SNPs is illustrated in table 1.

\section{Statistical Analysis}

Counting the number of genotyped loci identical for T2D risk alleles was performed to assess the possible cumulative recessive effects on traits related to the disease. In addition to assuming a recessive model, the number of total risk alleles was calculated to be able to test for additive effects on measured traits. We used Pearson's correlation to test for the association between continuous variables. If the distribution of a variable was skewed, we utilized Spearman's test. In both tests, the significance level was chosen at 0.05 , and all tests were two-tailed. Simple linear regression was utilized to measure effect levels, and multiple linear regression was utilized to assess the effect of multiple independent variables. Assessing environmental variation between subjects was conducted by calculating the daily caloric intake and the number of hours per week spent performing sedentary, moderate, and vigorous activities. A nutritional database was established to aid in the calculation of caloric values of dietary items consumed in Saudi Arabia.

\section{Results}

The statistical analyses performed on the collected sample are described in table 2 . The distribution of inbreeding coefficients is shown in table 3 . In this sample, about $63 \%$ of the participants reported having a history of parental consanguinity with varying degrees of relatedness. The proportion of parents who were reported to be first cousins was 
Table 2. Summary of all statistics

\begin{tabular}{llc}
\hline Variable & Mean \pm SD & Range \\
\hline Age, years & & \\
$\quad$ Patients $(\mathrm{n}=179)$ & $45.84 \pm 8.9$ & $23-61$ \\
$\quad$ Healthy participants $(\mathrm{n}=183)$ & $36.40 \pm 10.83$ & $18-71$ \\
Age at diagnosis, years $(\mathrm{n}=179)$ & $38.05 \pm 8.88$ & $20-57$ \\
FBG level, $(\mathrm{n}=154)$ & $95.47 \pm 23.89$ & $41-248$ \\
BMI ( $\mathrm{n}=183)$ & $28.15 \pm 6.858$ & $15.1-67$ \\
Waist circumference, cm $(\mathrm{n}=182)$ & $96.22 \pm 14.96$ & $60-151$ \\
Loci homozygous for T2D risk alleles, $\mathrm{n}$ & & \\
$\quad$ Patients ( $=157)$ & $14.80 \pm 2.96$ & $5-21$ \\
$\quad$ Healthy participants $(\mathrm{n}=159)$ & $15.18 \pm 2.66$ & $5-21$ \\
\hline
\end{tabular}

Table 3. Distribution of inbreeding coefficients in 177 families

\begin{tabular}{lrl}
\hline Inbreeding coefficient, \% & Frequency, $\mathrm{n}$ & Percentage \\
\hline 0 & 66 & 36.9 \\
0.001 & 8 & 4.5 \\
0.002 & 12 & 6.7 \\
0.004 & 9 & 5 \\
$0.024-0.044$ & 8 & 4.5 \\
$0.084-0.196$ & 4 & 2.25 \\
$0.39-1.5$ & 14 & 7.8 \\
$1.56-6.24$ & 24 & 13.5 \\
$6.25-12.5$ & 32 & 18 \\
\hline
\end{tabular}

$18 \%$. Further family information are given in detail in the online supplementary materials (for all online suppl. material, see www.karger.com/doi/10.1159/000362447).

A significant inverse association was detected between inbreeding coefficients and age at diagnosis (Spearman's coefficient: $-0.186, \mathrm{p}=0.013)$. The inverse association suggests that every increase in the inbreeding coefficient might be associated with a higher risk of developing the disease at a younger age. However, the categorization of the sample data according to a parental history of diabetes revealed a significant association in one group of patients (this is illustrated in fig. 2). The association between inbreeding coefficients and age at diagnosis in families which reported both parents as being affected is -0.502 $(\mathrm{p}=0.003)$. This higher correlation might indicate that those who have higher inbreeding coefficients and have both parents affected with T2D are assumed to develop the disease at a younger age compared to other individuals in the remaining groups (fig. 2).

Since we incorporated approximate values to account for tribal relatedness in calculating inbreeding coefficients,

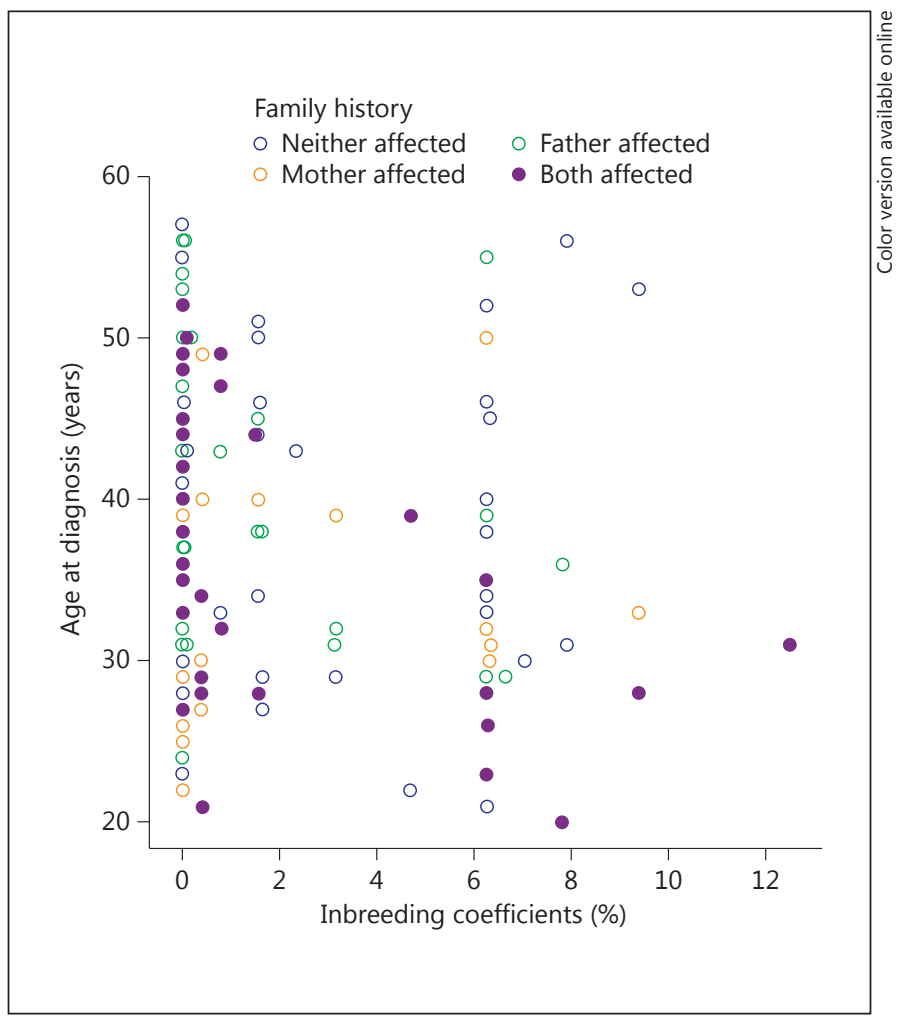

Fig. 2. Scatter plot of the correlation between age at diagnosis and inbreeding coefficients with the categorization of the sample data according to a parental history of T2D.

there could be a chance of introducing error. However, the contribution made by adding tribal relatedness information to calculated inbreeding coefficients is very small. To guard against possible errors caused by any invalid assumption, we assessed the association by calculating inbreeding coefficients without incorporating any tribal information. In this sensitivity analysis, we used only identified pedigree members to calculate inbreeding coefficients. Individuals whose parents were only related though a shared tribe or subtribe were presumed unrelated. The negative association between inbreeding coefficient and age at diagnosis remained statistically significant after removing tribal information (Spearman's coefficient: $-0.208, \mathrm{p}=0.005$ ).

No statistically significant associations were detected between inbreeding coefficients and BMI ( $\beta$ : 0.113, 95\% CI: -0.234 to 0.499$)$, waist circumference $(\beta: 0.207,95 \% \mathrm{CI}$ : -0.573 to 1.03 ) or FBG ( $\beta$ : $-0.007,95 \%$ CI: -1.16 to 1.15 ). (These associations remained statistically non-significant when removing tribal information from the calculated inbreeding coefficients.) However, in 42 families, we were able to recruit 2 healthy siblings. Pearson's correlation co- 


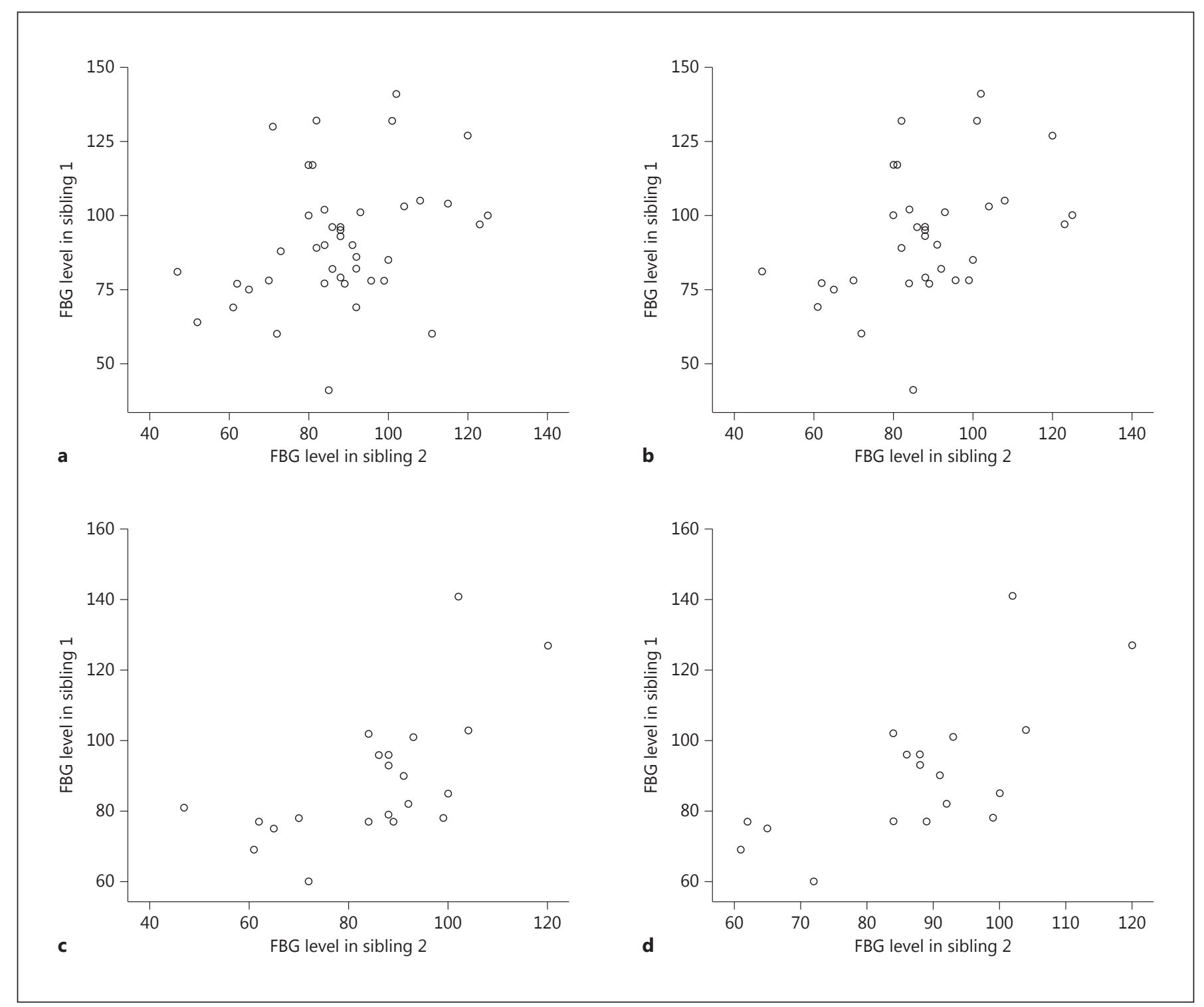

Fig. 3. Scatter plots illustrating the correlations of the FBG levels in families with different degrees of consanguinity. a All healthy siblings. b Siblings of all families with a history of consanguinity. c Siblings only of families with inbreeding coefficients $>0.04 \%$. d Siblings only of families with inbreeding coefficients $>1.5 \%$.

efficient of FBG levels between the siblings was 0.317 ( $\mathrm{p}=$ 0.04). To examine a possible effect of consanguinity, the correlations were calculated in 4 steps, each considering a more restricted range of consanguinity. The correlations between the siblings' FBG levels increased with an increasing degree of consanguinity as illustrated in table 4 and figure 3. This might indicate a stronger genetic influence induced by consanguinity leading to a smaller variation of FBG levels between siblings. The effect of consanguinity on the FBG level between siblings was assessed by a regression line analysis and controlling for differences in age, caloric intake, and level of physical activity $(\beta:-0.118, \mathrm{p}=0.024)$. Additionally, since all of the variables in this model are skewed, a log-transformation was applied, and the effect of consanguinity on the FBG level remained statistically significant $(\beta:-0.117, \mathrm{p}=0.038)$.

To observe possible recessive effects of the measured loci, we studied the associations between the number of loci identical for risk alleles and age at diagnosis, BMI, waist circumference, or FBG level. No significant associations 
Table 4. The effect of inbreeding on the correlation of FBG levels between siblings of 116 families

\begin{tabular}{lllc}
\hline & $\begin{array}{l}\text { Pearson's correlation } \\
\text { coefficient }\end{array}$ & Significance, $\mathrm{p}$ & Families, $\mathrm{n}$ \\
\hline All healthy siblings & 0.317 & 0.040 & 42 \\
Siblings only of families with a history of consanguinity & 0.419 & 0.015 & 33 \\
Siblings only of families with inbreeding coefficients $>0.04 \%$ & 0.584 & 0.003 & 23 \\
Siblings only of families with inbreeding coefficients $>1.5 \%$ & 0.699 & 0.001 & 18 \\
\hline
\end{tabular}

were detected (age at diagnosis - $\beta$ : $-0.106,95 \%$ CI: -0.796 to 0.584 ; $\mathrm{BMI}-\beta: 0.221,95 \% \mathrm{CI}:-0.340$ to 0.781 ; waist circumference $-\beta$ : $-0.278,95 \%$ CI: -1.517 to 0.690 , and FBG level $-\beta: 0.448,95 \%$ CI: -1.515 to 2.411 ). This might indicate that the measured SNPs did not provide any evidence for inbreeding depression on any trait related to T2D.

Additionally, we examined the association between the number of detected risk alleles (contributed from loci homozygous and heterozygous for the risk alleles). However, no significant association was detected between the overall number of risk alleles and other traits. Only one association between age at diagnosis and the total number of risk alleles became marginally significant after accounting for a parental history of diabetes and inbreeding coefficients $(\beta:-0.399, \mathrm{p}=0.052)$. The genetic association tests remained statistically non-significant after performing a sensitivity analysis for SNPs with strong linkage disequilibrium. Assuming that the association between consanguinity and the risk of T2D is genuine and not due to some confounder, this suggests that many more loci must contribute to the risk or that this study did not have sufficient power to detect an association with the SNPs selected.

\section{Discussion}

Our investigation was not able to find evidence of any recessive effects of the measured SNPs on the severity or risk factors of T2D. Any associations between the number of loci homozygous for the risk alleles and variables related to the severity or risk factors of the disease were not statistically significant. If there really is a causal association between inbreeding coefficients and the severity of $\mathrm{T} 2 \mathrm{D}$, then this suggests there may be many more T2D risk loci, all acting with weak recessive effects.

Although we had selected those 23 SNPs with the strongest marginal effects (detected through GWAS), our study lacked power to detect the cumulative evidence of these weak recessive effects. Given the achieved sample size, the study would have been able to detect correlation coefficients of 0.19 for the genetic association tests, assuming a two-sided type 1 error of $5 \%$ and $80 \%$ power to detect a true correlation. However, the effects detected for all questions were very small compared to the assumed effects. The detected effect sizes varied between -0.036 and 0.06 for the different components of the genetic association tests, where assumed coefficients varied between 0.16 and 0.2 . However, as there is an association between the inbreeding coefficients and age at diagnosis, this suggests many more recessively acting loci may contribute to the T2D risk.

The significant negative correlation between age at diagnosis and inbreeding coefficients suggests that a history of consanguinity might increase an individual's chance of developing the disease at a young(er) age. These findings are consistent with other studies suggesting an increased T2D risk with a higher degree of inbreeding [11-13]. However, these studies only measured the odds of the disease using consanguinity as a binary value. Our study developed a more in-depth analysis of the association between the continuous measurement of a history of consanguinity and other variables related to T2D while adjusting for environmental factors.

We might argue that offspring with a parental history of diabetes might have a higher chance of adopting a behaviour/attitude pushing them to investigate their blood glucose status earlier compared to those with no family history of the disease. This attitude might bias the association between consanguinity and age at diagnosis. This point might be valid but when investigating the association between age at diagnosis and an inbreeding history, we controlled for the effect of a parental history of the disease by dividing our subjects to 4 different groups. Therefore, the detected significant association between age at diagnosis and an inbreeding history in those subjects with both parents affected (Spearman's rank correlation: $-0.502, p=0.003$ ) is less likely to be biased by a tendency of siblings of parents with diabetes to be diagnosed with the disease at an earlier age. 
Our study detected a significant pairwise association of FBG levels between siblings $(r=0.317, p=0.040)$. Since we are comparing full siblings, this correlation could be attributed to similar inherited alleles (additive effects), to similar inherited genotypes (dominant effects) or to shared environmental factors. However, once we measured the correlation of FBG levels between siblings based on a limited range of inbreeding coefficients, we noticed an increase in correlation with increasing values of inbreeding coefficients.

An increased correlation of FBG levels with increasing values of inbreeding coefficients might indicate a dominant effect. Higher levels of inbreeding might increase the chance of inheriting similar alleles at particular loci. Additionally, even when we controlled for the effect of environmental variation between siblings, the effect of inbreeding coefficients on the variation of FBG levels was still significant $(\beta:-0.118, \mathrm{p}=0.024)$. The negative regression coefficient indicates that the difference between siblings' FBG levels is reduced with increased levels of inbreeding, even when the variance of the environmental effect on siblings is considered. Although, siblings with higher inbreeding coefficients are more likely to be genetically similar compared to others with lower coefficients leading to a higher similarity of their FBG levels, we cannot neglect that this genetic similarity is driven by having a higher chance of inheriting identical alleles at loci leading to possible cumulative recessive effects on the FBG levels.

The strengths of this study include the ability to utilize extended pedigrees with the introduction of new distant relatedness concepts which aided in the production of continuous values of inbreeding coefficients. Additionally, this study measured caloric intake and levels of physical activity to account for the effect of possible environmental variation on the FBG levels between siblings of each family. We were able to observe a significant effect of inbreeding to reduce the difference in FBG levels between sibling pairs accounting for the differences in physical activity and caloric intake in addition to the difference in age.

The main limitation of this study was the low power to detect recessive effects of genetic variants. Additionally, the inability to recruit female participants limits the generalizability of the recommendations of this study to the female population in Saudi Arabia. Another limitation was related to using age at diagnosis as a proxy for the development of T2D. It is almost impossible to accurately indicate age at development of T2D in the recruited subjects. This uncertainty is mainly caused by the nature of the disease, where on several occasions patients are asymptomatic or even when they are symptomatic they might not seek health advice promptly.

Potential Effect of Consanguinity on T2D

Susceptibility in a Saudi Population

Although we recruited T2D patients based on a diagnosis provided by their treating doctors, there might be a chance of misclassification of cases. We managed to avoid cases diagnosed as type 1 diabetes depending on medical diagnosis. However, due to the limited capability of PHCs in Saudi Arabia to diagnosis MODY (maturity onset diabetes of the young) cases, there might be a chance of misdiagnosing MODY cases as T2D, especially younger cases. Nonetheless, several SNPs were reported to be shared between MODY and T2D.

Based on this study's findings, we can suggest that those with affected parents who are related should be targeted when implementing secondary preventive measures. However, for this suggestion to be considered, further investigations are needed to replicate this association in other regions of Saudi Arabia. Furthermore, this study was restricted to males due to cultural barriers. Nonetheless, we can argue that the incidence of T2D is alike between genders and the causal pathways are likely to be the same in males and females.

Finding a statistically significant association between inbreeding coefficients and age at diagnosis, with a stronger association in patients where both parents are affected, might indicate the need for future research in specific subjects. We would argue that there is a need to target subjects with high degrees of consanguinity and a strong family history in further genetic investigations to examine the possible recessive effects of risk alleles. Similarly, finding larger correlations between FBG levels in subjects with higher levels of consanguinity might indicate that a similar approach of targeting healthy individuals with a high degree of consanguinity would increase the chance of detecting genetic markers with possible recessive effect on FBG levels.

References
1 IDF: IDF Diabetes Atlas Update 2012. http:// www.idf.org/diabetesatlas/5e/Update2012 (accessed 12/02/2013).

-2 Bacchus RA, Bell JL, Madkour M, Kilshaw B: The prevalence of diabetes mellitus in male Saudi Arabs. Diabetologia 1982;23:330-332.

3 Fatani HH, Mira SA, el-Zubier AG: Prevalence of diabetes mellitus in rural Saudi Arabia. Diabetes Care 1987;10:180-183.

4 Anokute CC: Epidemiologic studies of diabetes mellitus in Saudi Arabia - Part I - Screening of 3,158 males in King Saud University. J R Soc Health 1990;110:201-203.

5 El-Hazmi MA, Warsy AS, Al-Swailem AR, Al-Swailem AM, Sulaimani R, Al-Meshari AA: Diabetes mellitus and impaired glucose tolerance in Saudi Arabia. Ann Saudi Med 1996;16:381-385. 
-6 Al-Nuaim AR: Prevalence of glucose intolerance in urban and rural communities in Saudi Arabia. Diabet Med 1997;14:595-602.

7 Al-Nozha MM, Al-Maatouq MA, Al-Mazrou YY, Al-Harthi SS, Arafah MR, Khalil MZ, Khan NB, Al-Khadra A, Al-Marzouki K, Nouh MS, Abdullah M, Attas O, Al-Shahid MS, Al-Mobeireek A: Diabetes mellitus in Saudi Arabia. Saudi Med J 2004;25:1603-1610.

-8 Alqurashi KA, Aljabri KS, Bokhari SA: Prevalence of diabetes mellitus in a Saudi community. Ann Saudi Med 2011;31:19-23.

$\checkmark$ Elhadd TA, Al-Amoudi AA, Alzahrani AS: Epidemiology, clinical and complications profile of diabetes in Saudi Arabia: a review. Ann Saudi Med 2007;27:241-250.

10 Ntzani EE, Kavvoura FK: Genetic risk factors for type 2 diabetes: insights from the emerging genomic evidence. Curr Vasc Pharmacol 2012;10:147-155.

11 Bener A, Zirie M, Al-Rikabi A: Genetics, obesity, and environmental risk factors associated with type 2 diabetes. Croat Med J 2005;46: 302-307.

-12 Anokute CC: Suspected synergism between consanguinity and familial aggregation in type 2 diabetes mellitus in Saudi Arabia. J R Soc Health 1992;112:167-169.

-13 Bener A, Yousafzai MT, Al-Hamaq AO, Mohammad AG, Defronzo RA: Parental transmission of type 2 diabetes mellitus in a highly endogamous population. World J Diabetes 2013;4:40-46.

14 el-Hazmi MA, al-Swailem AR, Warsy AS, alSwailem AM, Sulaimani R, al-Meshari AA: Consanguinity among the Saudi Arabian population. J Med Genet 1995;32:623-626.

-15 El Mouzan MI, Al Salloum AA, Al Herbish AS, Qurachi MM, Al Omar AA: Consanguinity and major genetic disorders in Saudi children: a community-based cross-sectional study. Ann Saudi Med 2008;28:169-173.

-16 El-Mouzan MI, Al-Salloum AA, Al-Herbish AS, Qurachi MM, Al-Omar AA: Regional variations in the prevalence of consanguinity in Saudi Arabia. Saudi Med J 2007;28:18811884.

-17 Reiling E, van 't Riet E, Groenewoud MJ, Welschen LM, van Hove EC, Nijpels G, Maassen JA, Dekker JM, 't Hart LM: Combined effects of single-nucleotide polymorphisms in GCK, GCKR, G6PC2 and MTNR1B on fasting plasma glucose and type 2 diabetes risk. Diabetologia 2009;52:1866-1870.

18 JBS 2: Joint British Societies' guidelines on prevention of cardiovascular disease in clinical practice. Heart 2005. http://www.bcs. com/download/651/JBS2final.pdf (accessed November 10, 2011).

19 Falconer DS: Small populations: pedigreed populations and close inbreeding; in Falconer DS: Introduction to Quantitative Genetics, ed 2. New York, Longman Scientific and Technical, 1981, pp 75-93.

20 Whincup PH, Kaye SJ, Owen CG, et al: Birth weight and risk of type 2 diabetes: a systematic review. JAMA 2008;300:2886-2897.
Cauchi S, El Achhab Y, Choquet H, Dina C, Krempler F, Weitgasser R, Nejjari C, Patsch W, Chikri M, Meyre D, Froguel P: TCF7L2 is reproducibly associated with type 2 diabetes in various ethnic groups: a global meta-analysis. J Mol Med (Berl) 2007;85:777-782.

22 Dupuis J, Langenberg C, Prokopenko I, et al: New genetic loci implicated in fasting glucose homeostasis and their impact on type 2 diabetes risk. Nat Genet 2010;42:105-116.

23 Franklin CS, Aulchenko YS, Huffman JE, Vitart V, Hayward C, Polasek O, Knott S, Zgaga L, Zemunik T, Rudan I, Campbell H, Wright AF, Wild SH, Wilson JF: The TCF7L2 diabetes risk variant is associated with $\mathrm{HbAl}(\mathrm{C})$ levels: a genome-wide association meta-analysis. Ann Hum Genet 2010;74:471-478.

24 Saxena R, Voight BF, Lyssenko V, et al: Genome-wide association analysis identifies loci for type 2 diabetes and triglyceride levels. Science 2007;316:1331-1336.

25 Scott LJ, Mohlke KL, Bonnycastle LL, et al: A genome-wide association study of type 2 diabetes in Finns detects multiple susceptibility variants. Science 2007;316:1341-1345.

26 Steinthorsdottir V, Thorleifsson G, Reynisdottir I, et al: A variant in CDKAL1 influences insulin response and risk of type 2 diabetes. Nat Genet 2007;39:770-775.

27 Paré G, Chasman DI, Parker AN, Nathan DM, Miletich JP, Zee RY, Ridker PM: Novel association of HK1 with glycated hemoglobin in a non-diabetic population: a genome-wide evaluation of 14,618 participants in the Women's Genome Health Study. PLoS Genet 2008; 4:e1000312.

28 Lee YH, Kang ES, Kim SH, Han SJ, Kim CH, Kim HJ, Ahn CW, Cha BS, Nam M, Nam CM, Lee HC: Association between polymorphisms in SLC30A8, HHEX, CDKN2A/B, IGF2BP2, FTO, WFS1, CDKAL1, KCNQ1 and type 2 diabetes in the Korean population. J Hum Genet 2008;53:991-998.

29 Wu Y, Li H, Loos RJ, Yu Z, Ye X, Chen L, Pan $\mathrm{A}, \mathrm{Hu} \mathrm{FB}$, Lin $\mathrm{X}$ : Common variants in CDKAL1, CDKN2A/B, IGF2BP2, SLC30A8, and HHEX/IDE genes are associated with type 2 diabetes and impaired fasting glucose in a Chinese Han population. Diabetes 2008; 57:2834-2842.

30 Andersson EA, Pilgaard K, Pisinger C, Harder MN, Grarup N, Faerch K, Poulsen P, Witte DR, Jørgensen T, Vaag A, Hansen T, Pedersen O: Type 2 diabetes risk alleles near ADCY5, CDKAL1 and HHEX-IDE are associated with reduced birthweight. Diabetologia 2010;53: 1908-1916.

31 Zeggini E, Weedon MN, Lindgren CM, et al: Replication of genome-wide association signals in UK samples reveals risk loci for type 2 diabetes. Science 2007;316:1336-1341.

32 Nemr R, Almawi AW, Echtay A, Sater MS, Daher HS, Almawi WY: Replication study of common variants in CDKAL1 and CDKN2A/2B genes associated with type 2 diabetes in Lebanese Arab population. Diabetes Res Clin Pract 2012;95:e37-e40.
33 Lu F, Qian Y, Li H, Dong M, Lin Y, Du J, Lin Y, Chen J, Shen C, Jin G, Dai J, Hu Z, Shen H: Genetic variants on chromosome 6p21.1 and 6 p22.3 are associated with type 2 diabetes risk: a case-control study in Han Chinese. J Hum Genet 2012;57:320-325.

-34 Yasuda K, Miyake K, Horikawa Y, et al: Variants in KCNQ1 are associated with susceptibility to type 2 diabetes mellitus. Nat Genet 2008;40:1092-1097.

35 Unoki H, Takahashi A, Kawaguchi T, et al: SNPs in KCNQ1 are associated with susceptibility to type 2 diabetes in East Asian and European populations. Nat Genet 2008;40: 1098-1102.

36 Voight BF, Scott LJ, Steinthorsdottir V, et al: Twelve type 2 diabetes susceptibility loci identified through large-scale association analysis. Nat Genet 2010;42:579-589.

37 Been LF, Ralhan S, Wander GS, Mehra NK, Singh J, Mulvihill JJ, Aston CE, Sanghera DK: Variants in KCNQ1 increase type II diabetes susceptibility in South Asians: a study of 3,310 subjects from India and the US. BMC Med Genet 2011;12:18.

38 Takeuchi F, Katsuya T, Chakrewarthy S, et al: Common variants at the GCK, GCKR, G6PC2-ABCB11 and MTNR1B loci are associated with fasting glucose in two Asian populations. Diabetologia 2010;53:299-308.

39 Soranzo N, Sanna S, Wheeler E, et al: Common variants at 10 genomic loci influence hemoglobin $\mathrm{A}(\mathrm{C})$ levels via glycemic and nonglycemic pathways. Diabetes 2010;59:32293239.

40 Njolstad PR, Sagen JV, Bjorkhaug L, et al: Permanent neonatal diabetes caused by glucokinase deficiency: inborn error of the glucoseinsulin signaling pathway. Diabetes 2003;52: 2854-2860.

41 Hattersley AT, Ashcroft FM: Activating mutations in Kir6.2 and neonatal diabetes: new clinical syndromes, new scientific insights, and new therapy. Diabetes 2005;54:25032513.

42 Morgan AR, Thompson JM, Murphy R, Black PN, Lam WJ, Ferguson LR, Mitchell EA: Obesity and diabetes genes are associated with being born small for gestational age: results from the Auckland Birthweight Collaborative study. BMC Med Genet 2010;11:125.

43 Moller AM, Dalgaard LT, Ambye L, Hansen L, Schmitz O, Hansen T, Pedersen O: A novel Phe75fsdelT mutation in the hepatocyte nuclear factor-4alpha gene in a Danish pedigree with maturity-onset diabetes of the young. J Clin Endocrinol Metab 1999;84:367-369.

44 Horikawa Y, Iwasaki N, Hara M, Furuta H, Hinokio Y, Cockburn BN, Lindner T, Yamagata K, Ogata M, Tomonaga O, Kuroki H, Kasahara T, Iwamoto Y, Bell GI: Mutation in hepatocyte nuclear factor-1 beta gene (TCF2) associated with MODY. Nat Genet 1997;17: 384-385. 